\title{
Two Motion-Detection Algorithms for Projection-Reconstruction Magnetic Resonance Imaging: Theory and Experimental Verification
}

\author{
Rik Van de Walle ${ }^{1,2}$, Ignace Lemahieu ${ }^{1}$ and Eric Achten ${ }^{2}$ \\ 1 Department of Electronics and Information Systems, University of Ghent, \\ Sint-Pietersnieuwstraat 41, B-9000 Ghent, Belgium \\ 2 Department of Magnetic Resonance, Ghent University Hospital, \\ De Pintelaan 185, B-9000 Ghent, Belgium
}

\begin{abstract}
In this paper, projection-reconstruction (PR) magnetic resonance (MR) imaging is considered. We present two new quantitative techniques that allow to check when motion was present during a PR MR experiment. No a priori information about the motion is required since only the measured MR signals are used in the calculations. Moreover, the proposed methods can be implemented on a standard personal computer or workstation. It is experimentally shown that both methods are able to detect motion intervals with an accuracy of one repetition time.
\end{abstract}

\section{Introduction}

Magnetic resonance imaging has several advantages over other imaging techniques, including a high spatial resolution combined with a high soft-tissue contrast and its noninvasiveness. An important disadvantage of the technique is that workable images often require long measuring times compared with gross motion and physiological motion (e.g. blood flow, heart pulsation, peristaltic motion and breathing). The presence of motion during MR imaging results in a distortion of the measured MR signals. When images are reconstructed from these distorted signals, severe ghosting and blurring are often seen [1-5]. Several methods have been proposed to minimize these artifacts [6-15], but none of these is able to reduce artifacts caused by general motion completely. Therefore, the appearance of motion artifacts remains one of the major drawbacks in MR imaging.

\section{Theoretical Considerations}

The aim of two-dimensional (2D) MR imaging is to depict the 2D distribution of the complex transverse magnetization $\bar{M}(x, y)$ in a plane through a patient. In case of PR, a set of one-dimensional (1D) projections $\bar{P}_{\theta}(r)$ of $\bar{M}(x, y)$ is considered, where $\theta=0, \frac{1}{N_{P}} \pi, \frac{2}{N_{P}} \pi, \ldots, \frac{N_{P}-1}{N_{P}} \pi$ are the projection angles, $N_{P}$ is the total number of projections and $r$ is a place variable along the projection axis. 
When PR is used in MR imaging, a $1 \mathrm{D}$ signal $\bar{S}_{\theta}(k)$, consisting of $N_{S}$ samples in the spatial-frequency $k$-space, is measured for each projection angle $\theta$. The set $\left\{\bar{P}_{\theta}(r) ; \theta=0, \ldots, \frac{N_{P}-1}{N_{P}} \pi\right\}$ is related with the set $\left\{\bar{S}_{\theta}(k) ; \theta=0, \ldots, \frac{N_{P}-1}{N_{P}} \pi\right\}$ by a 1D Fourier transform with respect to $k$ :

$$
\bar{S}_{\theta}(k)=\int \bar{P}_{\theta}(r) e^{-j 2 \pi k r} d r .
$$

The projections $\bar{P}_{\theta}(r)$ can thus be calculated from the signals $\bar{S}_{\theta}(k)$ by performing an inverse Fourier transform on these signals and finally, the global distribution $\bar{M}(x, y)$ can be reconstructed from the set of projections by applying the filtered back-projection algorithm [16].

Generally, $\bar{P}_{\theta}(r)$ is a complex quantity since the transverse magnetization itself is complex. The signals $\bar{S}_{\theta}(k)$ are complex as well. However, from now on we consider amplitude images reconstructed by projection reconstruction. In this type of images, the transverse magnetization's magnitude $M(x, y)$ is depicted. Amplitude images can be reconstructed when the set of projection magnitudes $\left\{P_{\theta}(r) ; \theta=0, \ldots, \frac{N_{p}-1}{N_{P}} \pi\right\}$ is known (where $P_{\theta}(r)$ is the magnitude of the complex projection $\left.\bar{P}_{\theta}(r)\right)$, by applying the same filtered back-projection algorithm as mentioned before.

The projection magnitude $P_{\theta}(r)$ is a real number for each value of the place variable $r$ and the projection angle $\theta$. Therefore, the real part $\mathcal{R}_{\theta}(k)$ and the amplitude $A_{\theta}(k)$ of the measured signals $\left\{\bar{S}_{\theta}(k) ; \theta=0, \ldots, \frac{N_{p}-1}{N_{p}} \pi\right\}$ are even functions of $k$, while the imaginary $\mathcal{I}_{\theta}(k)$ part and the phase $\phi_{\theta}(k)$ of the signals are odd functions of $k$.

In practical $\mathrm{PR}$, the signals $\bar{S}_{\theta}(k)$ are sampled symmetrically around the origin $k=0$ for each $\theta=0, \ldots, \frac{N_{P}-1}{N_{P}} \pi$. By calculating the complex mean signal $\left\langle\bar{S}_{\theta}(k)\right\rangle_{k}$ with respect to $k$, we find

$$
\left\langle\bar{S}_{\theta}(k)\right\rangle_{k}=\left\langle\mathcal{R}_{\theta}(k)\right\rangle_{k} .
$$

Obviously, $\left\langle\widehat{S}_{\theta}(k)\right\rangle_{k}$ is real, which implies that its phase equals zero:

$$
\arg \left(\left\langle\bar{S}_{\theta}(k)\right\rangle_{k}\right)=0 .
$$

This conclusion holds as long as no motion is present during the measurement of $\bar{S}_{\theta}(k)$. Now it will be shown that it does not hold when motion is present during the MR experiment.

Indeed, it is well known that the presence of translational motion during an MR experiment results in a phase distorsion of the acquired signals. When the motion contains a rotational component, both the amplitude and phase of the signals are distorted. As a conclusion, when a general type of motion is present during the measurement of the signal $\bar{S}_{\theta}(k)$, the phase of $\bar{S}_{\theta}(k)$ will be distorted. Because of this phase distorsion, the mean value of the signal's imaginary part will differ from zero when motion is present during the measurement of $\bar{S}_{\theta}(k)$. As a result, the mean value $\left\langle\bar{S}_{\theta}(k)\right\rangle_{k}$ will not be a real number in this case and its phase will therefore differ from zero. 
From these considerations, a theoretical algorithm that allows to decide whether or not the studied object was moving during the measurement of the signal $\bar{S}_{\theta}(k)$, can be derived:

1. acquire $\bar{S}_{\theta}(k)$;

2. calculate $\arg \left(\left\langle S_{\theta}(k)\right\rangle_{k}\right)$;

3. decision:

- if $\arg \left(\left\langle\bar{S}_{\theta}(k)\right\rangle_{k}\right)=0 \rightarrow$ no motion occurred during the measurement of $\bar{S}_{\theta}(k)$;

- if $\arg \left(\left\langle\bar{S}_{\theta}(k)\right\rangle_{k}\right) \neq 0 \rightarrow$ motion did occur during the measurement of $\bar{S}_{\theta}(k)$.

In practical PR imaging, the measured signals are always noisy in some degree. As a result, the theoretically (i.e. when noiseless data were considered) derived Eq. 1 is never fulfilled exactly, even when the depicted object did not move during the MR experiment. Therefore, in order to be useful in practical situations, the proposed algorithm's decision step should be modified as follows:

3. decision:

- if $\left|\arg \left(\left\langle\bar{S}_{\theta}(k)\right\rangle_{k}\right)\right| \leq \alpha \rightarrow$ no motion occurred during the measurement of $\bar{S}_{\theta}(k)$;

- if $\left|\arg \left(\left\langle\bar{S}_{\theta}(k)\right\rangle_{k}\right)\right|>\alpha \rightarrow$ motion did occur during the measurement of $\bar{S}_{\theta}(k)$,

where $\alpha$ is a small critical phase, for which $\alpha=10^{\circ}$ is found to be a useful value when the $\arg \left(\left\langle\bar{S}_{\theta}(k)\right\rangle_{k}\right)$ values are calculated on a scale between $-180^{\circ}$ and $+180^{\circ}$ (see further).

In the next part of this section, a second method to determine motion intervals in projection-reconstruction magnetic resonance imaging will be explained. In a previous study, it was found that in case of translational motion with a frequency that is smaller than a critical frequency, the projections' amplitudes $P_{\theta}(r)$ are almost not distorted [3-5]. This critical frequency has a typical value of $50 \mathrm{~Hz}$.

As a result, when translational motion with small frequencies is present during a PR MR experiment, images without relevant motion artifacts can be reconstructed from the set $\left\{P_{\theta}(r) ; \theta=0, \ldots, \frac{N_{P}-1}{N_{P}} \pi\right\}$ by applying the filteredbackprojection algorithm.

Moreover, the root mean square deviation between two successive projections $P_{\theta}(r)$ and $P_{\theta-\pi / N_{P}}(r)$ will remain small when the considered object is not moving during the $M R$ experiment. This can be seen as follows. Consider the root mean square deviation between two consecutive projections:

$$
R M S D(\theta)=\sqrt{\sum_{r} \frac{\left(P_{\theta}(r)-P_{\theta-\pi / N_{P}}(r)\right)^{2}}{N_{P}}},
$$

where $\theta=\frac{1}{N_{P}} \pi, \ldots, \frac{N_{P}-1}{N_{P}} \pi$. 
Each projection $P_{\theta}(r)$ can be considered as a real function $P(\theta, r)$ of two variables, i.e. the projection-angle variable $\theta$ and the place variable $r$. By applying Taylor's expansion theorem [17] with respect to the projection-angle variable, the following expression can be written:

$$
\frac{\left(P_{\theta}(r)-P_{\theta-\pi / N_{P}}(r)\right)^{2}}{N_{P}}=\left(\frac{\delta P_{\theta}(r)}{\delta \theta}\right)^{2} \frac{\pi^{2}}{N_{P}^{3}}+\mathcal{O}\left[\frac{\pi^{3}}{N_{P}^{4}}\right]
$$

where $\mathcal{O}\left[\frac{\pi^{3}}{N_{P}^{4}}\right]$ denotes a term of order $\frac{\pi^{3}}{N_{P}^{4}}$. In practical situations, $N_{P}$ has a minimum value of 64 and therefore $\frac{\pi^{2}}{N_{P}^{3}}$ and $\frac{\pi^{3}}{N_{P}^{4}}$ will have a maximum order of magnitude of $4.10^{-5}$ and $2 \cdot 10^{-6}$ respectively. From Eq. 2 and 3 it can then be seen that $R M S D(\theta)$ will remain relatively small in this case. This conclusion holds as long as $\left(\frac{\delta P_{\theta}(r)}{\delta \theta}\right)^{2}$ remains small, which is only true when no motion or translational motion with a low frequency is present. When the object's motion contains $\mathrm{s}$ high-frequency translational or a rotational component during the measurement of the signal $\bar{S}_{\theta}(k), R M S D(\theta)$ becomes much larger since $\left(\frac{\delta P_{\theta}(r)}{\delta \theta}\right)^{2}$ becomes large in this case.

As a result, a second theoretical algorithm to determine whether the studied object moved during the measurement of $\bar{S}_{\theta}(k)$ can be derived:

1. acquire $\bar{S}_{\theta}(k)$;

2. calculate $R M S D(\theta)$;

3. decision:

- if $R M S D(\theta) \leq \beta \rightarrow$ no relevant motion occurred during the measurement of $\bar{S}_{\theta}(k)$;

- if $R M S D(\theta)>\beta \rightarrow$ motion did occur during the measurement of $\bar{S}_{\theta}(k)$,

where $\beta$ is a relatively small critical $R M S D$ value, for which $\beta=10$ is found to be a suitable value when the $R M S D(\theta)$ values are calculated on a scale in terms of percentage between 0 and 100 (see further). 'Relevant motion' should be interpreted as 'high-frequency translational motion or rotational motion', since low-frequency translational motion does not give rise to motion artifacts in PR imaging.

\section{Materials and methods}

In this study, sagittal images of a volunteer's brain were investigated. The corresponding MR signals were measured by using a PR sequence on a $1.5 \mathrm{~T}$ Siemens SP63 whole-body scanner. The images' field of view was $300 \mathrm{~mm}$, the total number of projections $N_{P}$ was 256 , the number of $k$-space samples per signal $N_{S}$ was 256 and the thickness of the image planes was $10 \mathrm{~mm}$. The PR sequence was characterized by an echo time of $20 \mathrm{~ms}$, a repetition time of $1000 \mathrm{~ms}$ and a readout time of $7.68 \mathrm{~ms}$. The signals $\bar{S}_{\theta}(k)$ were measured in algebraic order: the first signal corresponded with $\theta=0$, the second with $\theta=\frac{1}{256} \pi, \ldots$, the last with 
$\theta=\frac{255}{256} \pi$. A reference measurement was performed during which the volunteer was not moving.

In a first experiment, the volunteer's head was moving during two separate and long time intervals. These intervals corresponded with the measurement of the 17 th through the 41 st signal and the 114th through the 137th signal respectively. The head movement was continuous and contained both translational and rotational components. The maximum displacement in the direction perpendicular to the image plane was $2 \mathrm{~mm}$, while the maximum in-plane displacement was $45 \mathrm{~mm}$.

In the second experiment, the volunteer's head was moving in a similar way, but only during one short time interval, i.e. during the measurement of the 47 th trough 56th signal.

For each experiment, the intervals during which the volunteer was moving were determined from the measured sets of MR signals. This was done by calculating $R M S D(\theta)$ in terms of percentage on a scale between 0 and 100 and $\arg \left(\left\langle S_{\theta}(k)\right\rangle_{k}\right)$ on a scale between $-180^{\circ}$ and $+180^{\circ}$, for all projection angles $\theta=\frac{1}{256} \pi, \ldots, \frac{255}{256} \pi$. When $R M S D(\theta)$ was higher than $\beta=10$ or when the absolute value of $\arg \left(\left\langle\bar{S}_{\theta}(k)\right\rangle_{k}\right)$ was higher than $10^{\circ}$, it was decided that motion occurred during the measurement of $\bar{S}_{\theta}(k)$, according to the algorithms that were described previously.

\section{Results and discussion}

Figures 1 and 2 show the $R M S D(\theta)$ and $\arg \left(\left\langle\bar{S}_{\theta}(k)\right\rangle_{k}\right)$ values for the reference measurement. All $R M S M(\theta)$ and $\arg \left(\left\langle\bar{S}_{\theta}(k)\right\rangle_{k}\right)$ are lower than the critical values $\beta=10$ and $\alpha=10^{\circ}$ respectively, as was expected from the theoretical study.

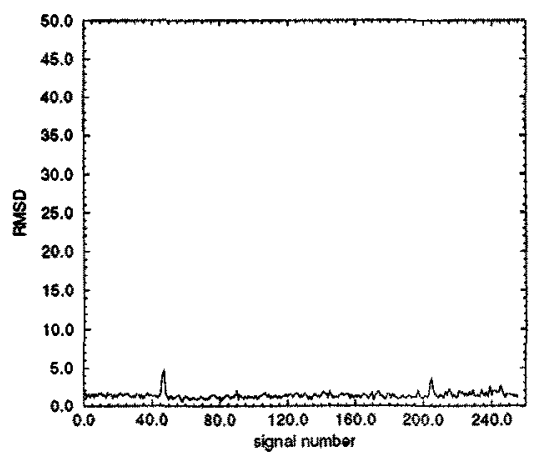

Fig. 1. Root mean square deviation as function of the signal number, corresponding with the reference image. 


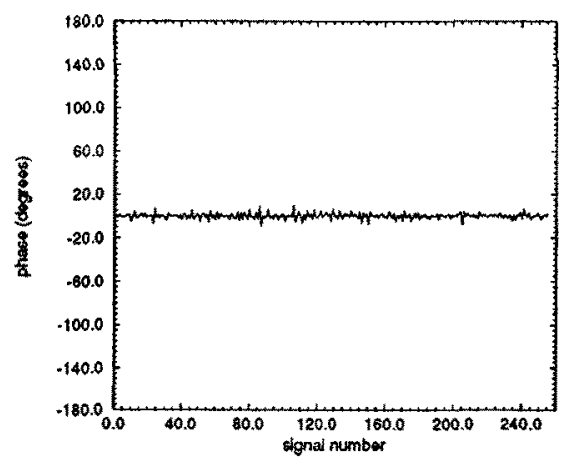

Fig. 2. Phase of the signals' complex means as function of the signal number, corresponding with the reference image.

The $R M S D(\theta)$ values that were found when motion occurred are shown in figures 3 and 4 , while the corresponding $\arg \left(\left\langle\bar{S}_{\theta}(k)\right\rangle_{k}\right)$ values are shown in figures 5 and 6 . Using the mentioned threshold $\beta=10$ for $R M S D(\theta)$, the motion intervals can be derived from figures 3 and 4 . For the first experiment, it is decided that motion occurred during the measurement of signals 17 through 40 and during the measurement of signals 113 through 137. In the second experiment, it is decided that motion was present during the measurement of signals 47 through 57.

The motion intervals can also be determined from figures 5 and 6 by using the threshold of $\alpha=10^{\circ}$. In this case, motion intervals are found by the motiondetection algorithm that correspond with the measurement of signals 17 through 40 and 115 through 138 for the first experiment, and 47 through 56 for the second experiment.

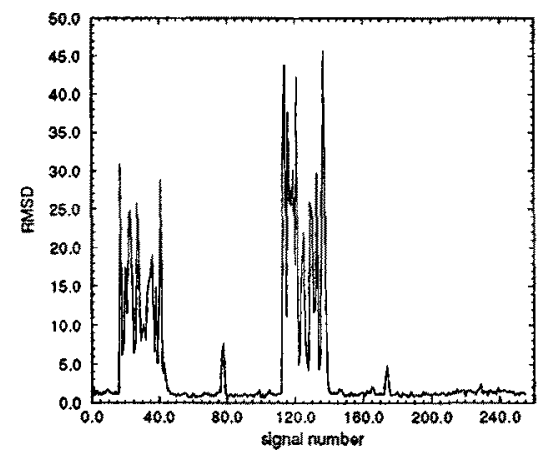

Fig. 3. Root mean square deviation as function of the signal number, in case of 2 long motion intervals. 


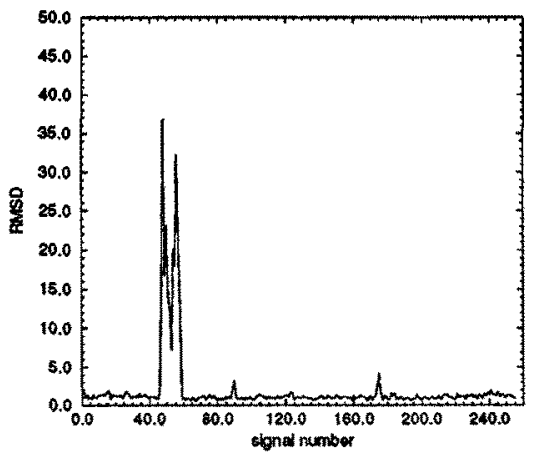

Fig. 4. Root mean square deviation as function of the signal number, in case of 1 short motion interval.

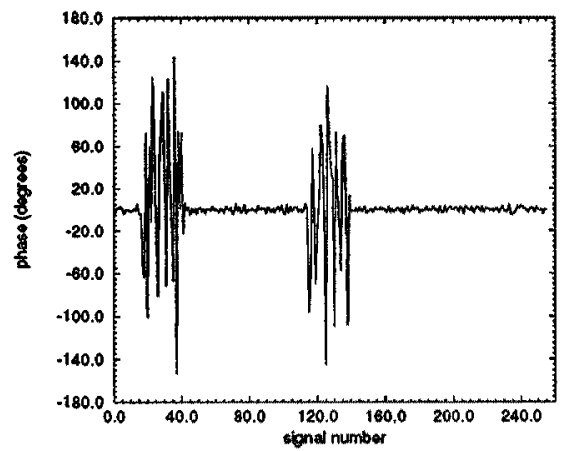

Fig. 5. Phase of the signals' complex means as function of the signal number, in case of 2 long motion intervals.

A summary of the results is given in table 1. Both motion-detection methods prove to be able to detect motion intervals in PR signals with high a accuracy: the maximum difference between the estimated intervals and the actual motion intervals is 1 signal number or 1 repetition time, both in case of long and repetitive motion (first experiment) and in case of short motion (second experiment).

\section{Conclusion}

We presented two new methods to detect motion intervals from a set of PR signals, based on a theoretical study. They do not require any a priori information on the motion and can be implemented easily. It was experimentally shown that 


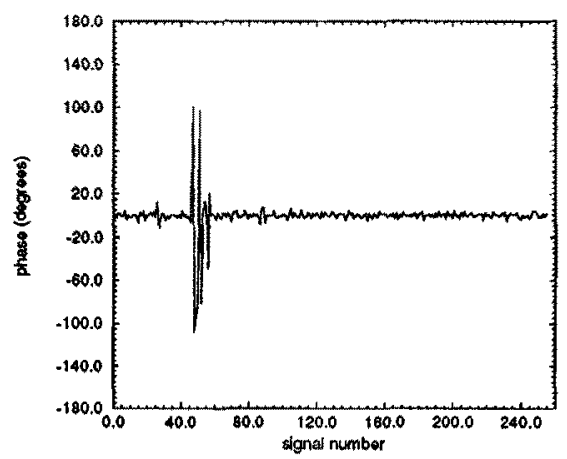

Fig. 6. Phase of the signals' complex means as function of the signal number, in case of 1 short motion interval,

\begin{tabular}{|c||c|c|c|}
\hline \hline & $\begin{array}{c}\text { real } \\
\text { motion intervals }\end{array}$ & $\begin{array}{c}\text { estimated } \\
\text { motion intervals } \\
R M S D(\theta)\end{array}$ & $\begin{array}{c}\text { estimated } \\
\text { motion interval } \\
\arg \left(\left\langle\bar{S}_{\theta}(k)\right\rangle_{k}\right)\end{array}$ \\
\hline 1st experiment & $17-41$ & $17-40$ & $17-40$ \\
& $114-137$ & $113-137$ & $115-138$ \\
\hline 2nd experiment & $47-56$ & $47-57$ & $47-56$ \\
\hline
\end{tabular}

Table 1. Summary of the experimental results.

both methods are able to detect these motion intervals with a high accuracy. This information can be used to remove the limited number of motion-corrupted signals from the set $\left\{\bar{S}_{\theta}(k) ; \theta=0, \ldots, \frac{N_{P}-1}{N_{P}} \pi\right\}$. Afterwards only this limited number of signals must be measured again to obtain a complete non-distorted set.

\section{Acknowledgement}

R. Van de Walle is supported by a $\mathrm{PhD}$ grant from the Flemish Institute for the Promotion of Scientific-Technological Research in the Industry (IWT, Brussels, Belgium). The experiments that are described in this paper were performed at the MR Department of the Ghent University Hospital (Belgium). The authors would like to thank the entire scientific and technical staff of this department for their support. 


\section{References}

1. Ehman, R.L., McNamara, M.T., Brasch, R.C., Felmlee, J.P., Gray, J.E., Higgins, C.B.: Influence of Physiologic Motion on the Appearance of Tissue in MR Images. Radiology 159 (1986) 777-782

2. Lauzon, M.L., Rutt, B.K.: Generalized K-Space Analysis and Correction of Motion Effects in MR Imaging. Magn. Reson. Med. 30 (1993) 438-446

3. Van de Walle, R., Voet, T., Lemahieu, I.: The Motion Sensitivity of Magnetic Resonance Imaging: A Comparison between Fourier Transform Imaging and Projection Reconstruction. Proceedings IEEE EMBC and CMBEC, 17th Conference, Montreal (1995) 457-458

4. Van de Walle, R., De Poorter, J., Voet, T., Lemahieu, I.: Quantitative Study of the Motion Sensitivity of Spin-Echo Imaging and Projection Reconstruction. Proceedings SMR, 3rd Annual Meeting, Nice (1995) 752

5. Van de Walle, R., Voet, T., Lemahieu, I.: Quantitative Analysis of the Motion Sensitivity of Projection Reconstruction in MR Imaging. Proceedings Medicon, 7 th Mediterranean Conference on Medical and Biological Engineering, Jerusalem, (1995) 180

6. Wood, M.L., Shivji, M.J., Stanchev, P.L.: Planar Motion Correction Using K-Space Data Acquired by Fourier MR Imaging. Proceedings SMR, 2nd annual Meeting; San Francisco (1994) 841

7. Dixon, W.T., Brummer, M.E., Malko, J.A.: Acquisition Order and Motional Artifact Reduction in Spin Warp Images. Magn. Reson. Med. 6 (1988) 74-83

8. Haacke, E.M.. Lenz, G.W.: Improving MR Image Quality in the Presence of Motion Using Rephasing Gradients. AJR 148 (1987) 1251-1258

9. Jung, K.J., Cho, Z.H.: Reduction of Flow Artifacts in NMR Diffusion Imaging Using View-Angle Tilted Line-Integral Projection Reconstruction. Magn. Reson. Med. 19 (1991) 349-360

10. Hedley, M., Yan, H.: Motion Artifact Suppression: A review of Post-Processing Techniques. Magn. Reson. Imaging 10 (1993) 627-635

11. Hedley, M., Yan, H., Rosenfeld, D.: Motion Artifact Correction in MRI Using Generalized Projections. IEEE Trans. Med. Imaging 10 (1991) 40-46

12. Lui, J, Koenig, J.L.: An Automatic Phase Correction Method in NMRI. J. Magn. Reson. 86 (1990) 593-604

13. Zang, L.H., Fielden, J., Wilbrink, J., Takane, A., Koizumi, H.: Correction of Translational Motion Artifacts in Multi-Slice Spin-echo Imaging Using Self-Calibration. Magn. Reson. Med. 29 (1993) 327-334

14. Gmitro, A.F., Alexander, A.L.: Use of a Projection Reconstruction Method to Decrease Motion Sensitivity in Diffusion-Weighted MRI. Magn. Reson. Med. 29 (1993) 835-838

15. Glover, G.H., Pauly, J.M.: Projection Reconstruction Techniques for Reduction of Motion Effects in MRI. Magn. Reson. Med. 28 (1992) 275-289

16. Herman, G.T.: Image Reconstructions from Projections, Implementations and Applications, Springer Verlag, Berlin (1979)

17. Arfken, G.: Mathematical Methods for Physicists - 3rd Edition, Academic Press, San Diego (1985) 